\title{
Chapter 3 \\ A Haptic-Based Virtual Reality Head and Neck Model for Dental Education
}

\author{
Paul Anderson, Minhua Ma and Matthieu Poyade
}

\begin{abstract}
There have been numerous datasets, 3D models and simulations developed over the years however it is clear that there is a need to provide an anatomically accurate, flexible, user driven virtual training environment that can potentially offer significant advantages over traditional teaching methods, techniques and practices. The ability to virtually train dental trainees to navigate and interact in a repeatable format, before directly engaging with the patients can measurably reduce error rates while significantly enhancing the learner experience. Accurate dental simulation with force feedback allows dental students to familiarize with clinical procedures and master practical skills with realistic tactual sensation. In this chapter, we review the state of art of using haptics in dental training and present the development and construction of a medically validated high-definition interactive $3 \mathrm{D}$ head and neck anatomical dataset with a haptic interface to support and enhance dental teaching across multiple training sites for NHS Education Scotland. Data acquisition from cadaveric specimens and 3D laser scanning of precision dissection is discussed, including techniques employed to build digital models capable of real-time interaction and display. Digital anatomical model construction is briefly described, including the necessity to clinically validate each stage of development that would ensure a normalised human data set whilst removing anatomical variance arising from individual donor cadaveric material. This complex digital model was transformed into a real-time environment capable of largescale 3D stereo display in medical teaching labs across Scotland, whilst also offering the support for single users with laptops and PC. The 3D viewer environment also
\end{abstract}

\author{
P. Anderson · M. Ma $(\bowtie) \cdot$ M. Poyade \\ Digital Design Studio, Glasgow School of Art, Glasgow, UK \\ e-mail:m.ma@gsa.ac.uk \\ P. Anderson \\ e-mail: p.anderson@gsa.ac.uk \\ M. Poyade \\ e-mail:m.poyade@gsa.ac.uk
}


supports haptic interaction through a force feedback probe device (Phantom Omni) offering the ability for users to repeatedly practise giving dental anaesthesia injections into the gum. Specific tools supported include guillotine tools, picking and selection tools capable of expanding specific local regions of anatomy. Zoom camera functions and freeform rotation allows thorough and meaningful investigation to take place of all major and minor anatomical structures and systems whilst providing the user with the means to record sessions and individual scenes for learning and training purposes.

\subsection{Introduction}

As it is already routinely applied to many scientific fields, 3D scanning technology, including laser scanning and white light scanning, are now being used to explore applications in medicine. In healthcare, the technology has also been used in development of prostheses [1]. Translated scan data can be immediately usable in computer aided design software, improving the speed of development of prosthesis. One of the limitations of laser scanning technology is that it is only able to capture and reconstruct the outer surface of the body, therefore the scans do not have any internal structure and physical properties regarding skeleton, skin or soft tissues of the scanned human body, unless it is combined with cadaveric dissection [2].

On the other hand, medical visualization based on direct and indirect volumetric visualization uses data derived from 3D imaging modalities such as Computed Tomography (CT), Magnetic Resonance Imaging (MRI), cryosection images, or confocal microscopy [3]. Although, the visualization is generally accurate, it only represents a particular human body or cadaveric specimen. Demonstrating a normalised anatomically correct model is difficult due to the source of data, the largely elderly population of cadaveric specimens. In indirect volume visualization where individual surface models are reconstructed, mistakes and inaccuracy might be introduced from the manual or automatic segmentation process; whereas in direct volumetric visualization, interactivity is limited since surface geometries are not reconstructed [3]. As a result, the users are not able to manipulate the model (volumetric data) as they could on surface models. Functions such as virtual dissection, e.g. disassemble/reassemble, and studying individual substructures are not possible. Furthermore, each imaging modality has its limitations, for example, for cryosections, the cadaver had to be segmented into large blocks which results in a loss of data at certain intervals; for CT/MRI images, segmentation rarely focuses on very thin anatomic structures [4] such as fascia. Developing a model that can present thin anatomic structures would be of great interest to medical professionals and trainees. However, the ability to accurately segment very thin structures is a challenging and substantial task.

In this chapter we present an established workflow using state-of-the-art laser scanning technology and software for design and construction of 3D medical data, and describe the workflow practices and protocols in the Head and Neck Anatomy 


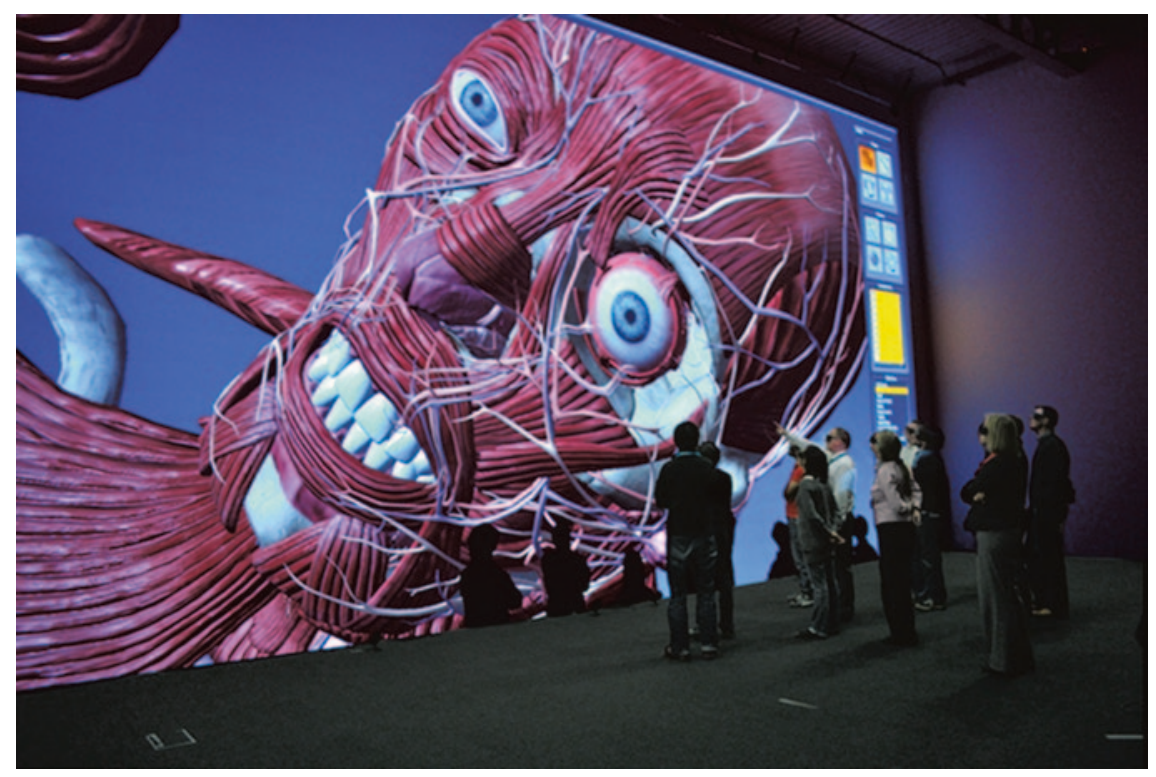

Fig. 3.1 The head and neck system was presented in the large scale virtual reality laboratory

project at the Digital Design Studio (DDS). The workflow overcomes the above limitations in volumetric visualization and surface anatomy.

This work was conducted by a well-established, unique, multi-disciplinary team drawn from art, technology and science. The team includes computer scientists, 3D modelers, mathematicians, artists and product designers, and supports a culture of research and creativity which is fast moving, highly productive, externally engaged and autonomous. This successful academic hybrid model is built upon strong collaborative partnerships directly with industry and end users, resulting in tangible real-world outputs. We have sought to establish a balanced portfolio of research, teaching and commercialization operating within a state-ofthe-art, custom built facilities located within the heart of the Digital Media Quarter in Glasgow. For instance, the DDS houses one of the largest virtual reality and motion capture laboratories in the world ensuring that we are at the forefront of digital innovation, developing products focused on immersive virtual environments, highly realistic digital 3D models and prototypes, user interfaces and avatars. The virtual reality laboratory enables 30-40 users to simultaneously experience real-time and interactive simulations as shown in Fig. 3.1.

This haptic-based 3D models of head and neck anatomy has been used to educate health professionals and to support activities such as pre-operative procedure planning and surgical simulation in order to improve risk management and thus increase patient safety. This work has recently received significant recognition in the RCUK report Big Ideas for the Future [5]. 


\subsection{Haptics in Dental Training}

In dental education, specific training needs to be conducted to allow dentist apprentices and dental hygienists to acquire the psychomotor skills that are required to achieve complex clinical procedures such as anaesthetic injection, oral and maxillofacial restorative surgery, and dental implant preparation [6]. Traditionally, that training occurs under the supervision of dental experts and is carried out on plastic replicas of human jaws or live patients [6-9, ]. However, that training is limited to the availability of experts for supervision [10] and its effectiveness is mainly established on the basis of a subjective assessment of performance outcomes $[11,12]$. Moreover, training on replica model is often considered to not to be challenging enough $[6,10]$ and lacks of tactile realism of human tissues $[9,12]$. On the other hand, training on live patients has been shown to be a source of anxiety for novice dental students [13, 14] and may eventually be critical from an ethical perspective [8].

Virtual Reality (VR) simulations allow solving those issues that arise throughout the training conventionally carried out in dental education [6,8-11]. An important concept of VR simulations to support the development of psychomotor skill in dental education is the haptic interaction [15]. Effectively, graphic and haptic cues are considered to complement each other throughout VR simulations [7].

The term haptic refers to the sense of touch. Haptic interfaces enable providing realistic tactile and kinesthetic feedback allowing the simulation of tactile sensations that are crucial throughout dental procedures [16], for instance the stiffness and friction properties of human tissues $[6,9,11,15]$.

During these last years, dedicated research efforts have been made to develop challenging applications of VR enhanced with haptics to improve the acquisition of psychomotor skills during training in dental education $[6,8,17]$. Some of those applications have been validated and are nowadays part of the dental curriculum of colleges of dentistry across the USA and the EU $[6,8,18]$.

This section proposes an overview of most successful applications that have been developed recently. Most of these developments propose cost effective solutions for the implementation of haptic interaction in virtual environments employing punctual inter-actuator such as computer gaming haptic devices from Novint Technologies (http://www.novint.com/) [12] or low cost interfaces from the Phantom product of Sensable Technologies Inc. (http://www.geomagic.com/en/) $[6,8,18]$. Most of these devices, in the proposed setup, are able to sense hand's position and orientation onto 6 Degrees Of Freedom (DOF), but can only provide force feedback onto 3DOF. In most developments, this has been highlighted as a limitation to the fidelity of the simulated tactile sensations. Moreover, the haptic rendering did not consider multi-point collision detection and single point-based collision was performed with regards to volumetric data set which often implicated a high computational cost [6] when no optimization was implemented (e.g. BSP Tree, octree data structure).

The HAP-DENT system [19], was presented as a promising educational tool for dental procedures [20]. It enabled practicing tooth drilling operations on a virtual jaw model displayed on a bi-dimensional monitor. The system was 
heuristically parameterized allowing defining a realistic force feedback model using a custom-made force measurement system. So far, no validation study on dental apprentices has been conducted to assess the effectiveness of the system to support psychomotor skill acquisition.

An original development of oral surgery simulator, known as the Forsslund system, was presented in [21]. It consisted of a working prototype to practice teeth drilling using a haptic device which computed volumetric tooth data and a semi-transparent planar mirror setup on which an active stereoscopic monitor was reflected allowing thus to visually match the haptic workspace with that of the virtual environment. The effectiveness of the suggested training tool to improve dental education has been highlighted in an evaluation study [22]. This system has been recently incorporated to the training curriculum of the Academic Centre for Dentistry Amsterdam (ACTA), Netherlands [8].

The PerioSim $\odot[16,23]$ was a highly realistic training platform which aimed to improve skills on diagnosis and treatment of periodontal diseases. It included the stereoscopic visualization of a jaw data model and supports haptic interaction. The haptic device enabled the manipulation of the probes that are necessary for the realization of periodontal tasks. A previous evaluation study has highlighted the potential of the PerioSim@ $\odot$ for educational purpose [7]. It is currently employed as part of the dental training curriculum of the University of Illinois in Chicago.

The HapTEL [12] consists of a training and assessment platform which supports physical practices of caries removal for which drilling operations are commonly carried out. As in [21], it presented a setup in which the haptic and visual workspaces were visually matching. However, the trainee's point of view was tracked so that visuo-haptic matching remains more accurate than that proposed in [21]. The originality of that development was that the haptic device did not model the training instrument but the deformation of the drilled tooth tissues. In fact, the trainee handled a real drilling instrument attached to the sensed tip of the haptic device.

Initially thought for bone drilling simulations [24], the Voxel-Man Dental simulators supported training on drilling operations commonly performed previously to dental restorative treatments. Alike the developments presented above, it allowed to stereoscopically display a virtual mouth model but also enabled bi-manual haptic interaction. A virtual drilling tool was handled in one hand and another instrument in the other (http://www.voxel-man.com/simulator/dental/). The concept of Voxel-Man simulators have been through a complete validation process enabling to be currently a successfully distributed training platform which allows generating subtle and convincing tactile differences between tissues [18].

Similarly, Suebnukarn et al. [25] proposed a haptic-based VR crown preparation simulator on isolated teeth enhanced with bi-manual haptic interaction. The proposed system enables assessing psychomotor skills in real-time and providing augmented feedback to support the successful development of the psychomotor skills required in such restorative operations [26]. An advanced version of the development proposed the implementation of the training principles in an Augmented Reality (AR) environment enhanced with similar haptic paradigm [27].

The Simodont Dental trainer [28] by Moog Inc. (http://www.moog.com/) consists of an educational tool to support training of drilling operations. Evaluation 
has demonstrated that Simodont Dental trainer has similar effectiveness to improve psychomotor skills than the training traditionally carried out on anatomical mock-up. As the development presented in $[12,21]$, the system used a mirror setup to support the stereoscopic visualization of volumetric teeth data but used an admittance haptic device that similarly, provided force feedback onto 3 DOF. Alike the Forsslund System [21], the Simodont Dental trainer has been also incorporated to the training curriculum of ACTA [8].

The VirDentT $[8,29]$ is prosthodontal training and assessment tool enhanced with haptic interaction. Alike the Voxel-Man Dental, It allows practicing restorative dental preparation tasks for fixed dental prostheses. It simulated a virtual drill along with a patient jaw. However, little information is available concerning technical details of the system and more investigation is needed before formally considering it as an effective training platform.

A haptic-based dental simulator, the iDental [30], consists of a training system at an early stage of development and evaluation. It currently supports the learning of psychomotor skills involved in periodontal procedures but aims to be extended to endodontal and prosthodontal operations. Training is carried out on a virtual mouth model in which symptoms of periodontal inflammations are shown. A haptic device is used to handle virtual probes needed to complete the treatment. The evaluation study presented in Wang et al. [30] suggested the construct validity of the system although the fidelity of graphical and haptic cues still needs to be improved.

Despite the many research efforts realized in order to improve training curriculum in dental education, it has not been reported so far, haptic-enhanced VR simulations to support training in pre-clinical procedures such as the inferior alveolar and lingual nerve block anaesthesia injection technique. Closest approaches considered an anatomical jaw model made of latex, which attempted emulating intrinsic haptic sensations perceived during the realization of the task in the real world, while practicing in an immersive virtual environment [31]. With regards to VR simulations of epidural injections, catheter insertion, needle biopsy lumbar puncture and virtual acupuncture presented during these last years in order to improve medical training $[32,33]$, VR enhanced with haptics is expected to play a growing role in the training curriculum of pre-clinical pinching procedures in dental education [34, 35].

\subsection{Development of Head and Neck Anatomy}

NHS Education Scotland (NES) launched a European tender to develop a four strand work package to develop digital content for interactive Head and Neck anatomy, instrument decontamination, virtual patients and common disease processes for dentistry. This was a complex project requiring high levels of interaction across multidisciplinary development partners, in order to build a digital anatomy model (the 3D Definitive Head and Neck) and other digital products for medical teaching, across a distributed network of centres and primary care settings. The research took place within established protocols concerned with government 
legislation and patient interaction where the security of data and appropriate interface development were key factors.

This chapter discusses and focuses on the Work Package A, i.e. the 3D interactive Head and Neck Anatomy. The aim of this project, commissioned by NES, was to complete the construction of the world's most accurate and detailed anatomical digital model of the head and neck using state-of-the-art data acquisition techniques combined with advanced, real-time 3D modelling skills and interactive visualization expertise. It was felt essential that this digital model must be capable of realtime interaction supporting both medical training and personal exploration, with all 3D data models and information being fully annotated, medically validated, and interactively "disassembled" to isolate and study individual substructures then reassembled at the touch of a button. In order to create a truly accurate interactive digital 3D model of head and neck anatomy it was essential to base the models upon real data acquired from both cadaveric and live human subjects. The full range of digital products was formally launched in April 2013. User feedback from NES medical teaching centres and primary care settings has been extremely positive.

This model integrates different tissue types, vasculature, and numerous substructures that are suitable for both the casual user and, in particular, those engaged in medical learning and teaching. Our model and software interface provides fluid and intuitive ways to visualise and encourage meaningful engagement with human anatomy amongst diverse audiences and is currently being well received by medical trainees, clinicians and the general public. Our 3D digital model development process, including data acquisition, model construction, interface design and implementation has been critically evaluated and validated by multi-disciplinary experts in the fields of medicine and computing science. Our extensive collaborative research network includes senior clinicians, surgical consultants, anatomists and biomedical scientists within the NHS, formal links with the medical schools within the Universities of Glasgow, Edinburgh, Dundee, Manchester and London, and other key specialists in the Scottish Medical Visualization Network.

Our systematic review of the medical visualization landscape indicated that there was an unmet need for validated anatomical visualizations of the healthy human body that could be relied upon by viewers as accurate and realistic reproductions of the real thing. Indeed, within medical, dental and surgical curricula, the number of actual contact hours for teaching has markedly reduced over recent years. Within the medical curriculum alone, the General Medical Council issued guidelines to medical schools in the United Kingdom (Tomorrow's Doctors, 1993) requesting a reduction in the amount of factual information [36]. This has happened across many medical schools across the world [36-38]. However, medical training programs also began to change to a more integrated curriculum with various teaching methodologies adopted. More recently in the UK, Tomorrow's Doctors 2009 has placed more emphasis on the medical sciences related to clinical practice. This directly reflects opinion from academics, clinicians and students that the anatomy content had been significantly "dumbed down" previously [39].

Thankfully, this is changing within medical, dental and surgical curricula. Interestingly, with these changes, it has also been shown that to optimise learning, 
Fig. 3.2 Development workflow


a variety of teaching modalities need to be used alongside traditional techniques. There is now an increased demand from anatomical educators for additional teaching resources, including those of a virtual nature and involving interactive multimedia [40-42]. In addition, the general public has no means to view and interact with a truly representative visual simulation of the human body in a way that provides a genuine educational experience to promote public understanding of health and wellbeing. Using visualization experience gained within the automotive, defense and built environment sectors alongside medical visualization research, we sought to address these shortfalls by constructing a high fidelity 3D dataset, supporting meaningful user engagement, viewing and real-time interaction.

The primary focus of the virtual head and neck anatomy is on user interaction with real-time digital data that supports multi-disciplinary skill sets. It is built upon through 3D laser scanning, 2D data capture, data processing and optimisation, 3D construction of objects and environments, photo-realistic rendering, user interface design, real-time display and cross-platform development.

Figure 3.2 shows the development workflow, which at a high level, consists of identification of a suitable donated cadaver, dissection, 3D laser scanning capturing surface measured data, 3D computer modelling of all structures, digital photography from surgical procedures, texture mapping (colour information onto 3D surfaces) and interface development to support user interactions, trials and testing. Verification and validation is conducted at every development stage with final results being presented to a clinical advisory panel who met every three months throughout the project period.

\subsubsection{Data Construction}

An important consideration with a project of this size is the sustainability of the workflow, tools and datasets that are generated over its duration. One of the dangers of working within the computing industry is that software and proprietary data formats can often become obsolete over time resulting in data that cannot be read or used. Consequently, it is good practice to ensure that any valuable data is stored using standardized, open and well-documented formats to ensure that it is not reliant on a 
single company or piece of software to be used. We adopted such an approach for the storage and preservation of the head and neck anatomical datasets. The data generated throughout this project can be separated into two groups. The first group encompasses the raw data such as photographic evidence and point cloud data generated from white light and 3D laser scanners. The second group includes the processed data created by the DDS modelling team. The non-specialist nature of the raw data enables us to store it using open file formats such as the Portable Network Graphics (PNG) format for photographs and images and the Stanford Triangle Format (PLY) for scan data. However, in order to process this data and create anatomical models, more specialist proprietary tools and data formats are required. Since this cannot be avoided, we used industry standard tools such as Autodesk's Maya and Pixologic's ZBrush to generate and store these models. In order to ensure long-term sustainability, the textured meshes are also exported and stored using an open format such as COLLADA to insure against any of these products becoming obsolete in the future.

\subsubsection{Data Acquisition}

We have developed a completely new approach to medical visualization. Our novel data construction workflow and validation process uses donor cadaveric material and through the process of destructive dissection and staged, high resolution laser scanning allows us to produce an accurate 3D anatomical model of head and neck anatomy. To ensure accuracy and a true likeness of the human body, this dataset was validated by the project's Clinical Advisory Board, which comprises anatomists, clinicians and surgical specialists. Our dataset and interface development focuses on real-time interactive simulation (not pre-rendered animations) which allows users to fully explore the complete anatomical structure and investigate in 3D at any scale, from a laptop or mobile device to a fully immersive environment (see Fig. 3.1).

\subsubsection{Selection of Specimen}

The identification of a suitable embalmed male Caucasian cadaver between the ages of 50-65 was the starting point for this work package. This was identified from the regular stock in the Laboratory of Human Anatomy, School of Life Sciences, College of Medical, Veterinary and Life Sciences at the University of Glasgow. The cadaver had no obvious signs of pre-existing craniofacial abnormalities. All procedures were carried out under the Anatomy Act 1984 and the Human Tissue (Scotland) Act 2006. This was undertaken by the government's Licensed Teacher of Anatomy. This formed the basis of the definitive 3D digital human, with the head and neck region comprising the element to be described. Minimal pre-existing age-related changes were present, significant because the majority of cadavers are of those who have died in old age, many of whom are edentulous. The alveolar bone and teeth were recreated digitally based on laser scans of disarticulated teeth, held at Glasgow Dental School. 


\subsubsection{Dissection and Data Capture of Head and Neck Soft Tissue}

The high resolution 3D laser scanning supported by high-resolution colour imaging capture was performed on the cadaver before formaldehyde embalming. The Perceptron Scanworks V5 3D laser scanner was used to capture accurate data of the surface geometry. Intra-oral scanning was also performed prior to the preservation process to allow accurate reconstruction of dental related anatomy (and to establish key anatomical and clinically relevant key landmarks) when there is pliability/mobility at the temporomandibular joint. The embalming procedure was carried out through collaboration of a mortician and qualified embalmer, supervised by a Licensed Teacher of Anatomy in the Laboratory of Human Anatomy, University of Glasgow. The eyes were injected with a saline solution postembalming to maintain its life-like contour, a technique established in anatomical and surgical training for ocular (and ocular related) surgical procedures designed by the Canniesburn Plastic Surgery Unit, an international leader in plastic and reconstructive training, research and clinical expertise.

Skin and subcutaneous tissue were meticulously dissected (using standard anatomical techniques) from the head and neck territories with appropriate health and safety precautions typical in the environment of using cadaveric tissue. Superficial muscles, nerves, glands and blood vessels were identified. Scanned muscles and attachments included the sternocleidomastoid, infrahyoid muscles, muscles of facial expression (including those around and within the eyes and nose) and the superficial muscles of mastication, including masseter and temporalis, all of which have important clinical and functional applications. The superficial nerves captured at this stage were the major sensory and motor innervations of the head and neck including the trigeminal and facial nerves, and specifically the termination of the facial nerve onto the muscles of facial expression. Scanned glands included the major salivary glands, i.e. the parotid, submandibular and the sublingual glands, as well as the endocrine thyroid gland. The blood vessels identified at this stage are the facial vessels as well as the jugular venous drainage of superficial anatomical structures.

Deeper dissection of the head included data capture for the training of oral related musculature including genioglossus, geniohyoid, mylohyoid, lateral and medial pterygoids, digastric and buccinators amongst others. These specific muscles are significantly important in oral function, and have immense clinical importance for dental training. The related nerve and blood supply to these structures were captured as previously described.

Neck dissection (down to the thoracic inlet) proceeded deeper to identify and capture major and minor structures at this site. Blood vessels (and related branching) were meticulously dissected to demonstrate arterial supply and venous drainage including the common carotid, subclavian and brachiocephalic trunk. Venous drainage includes internal jugular and subclavian veins, and all tributaries. The relationship of these blood vessels to important nerve structures in the neck demonstrated the close proximity to other structures and included the vagus and phrenic nerves, sympathetic trunk and the brachial plexus in the neck (supplying motor and sensory 


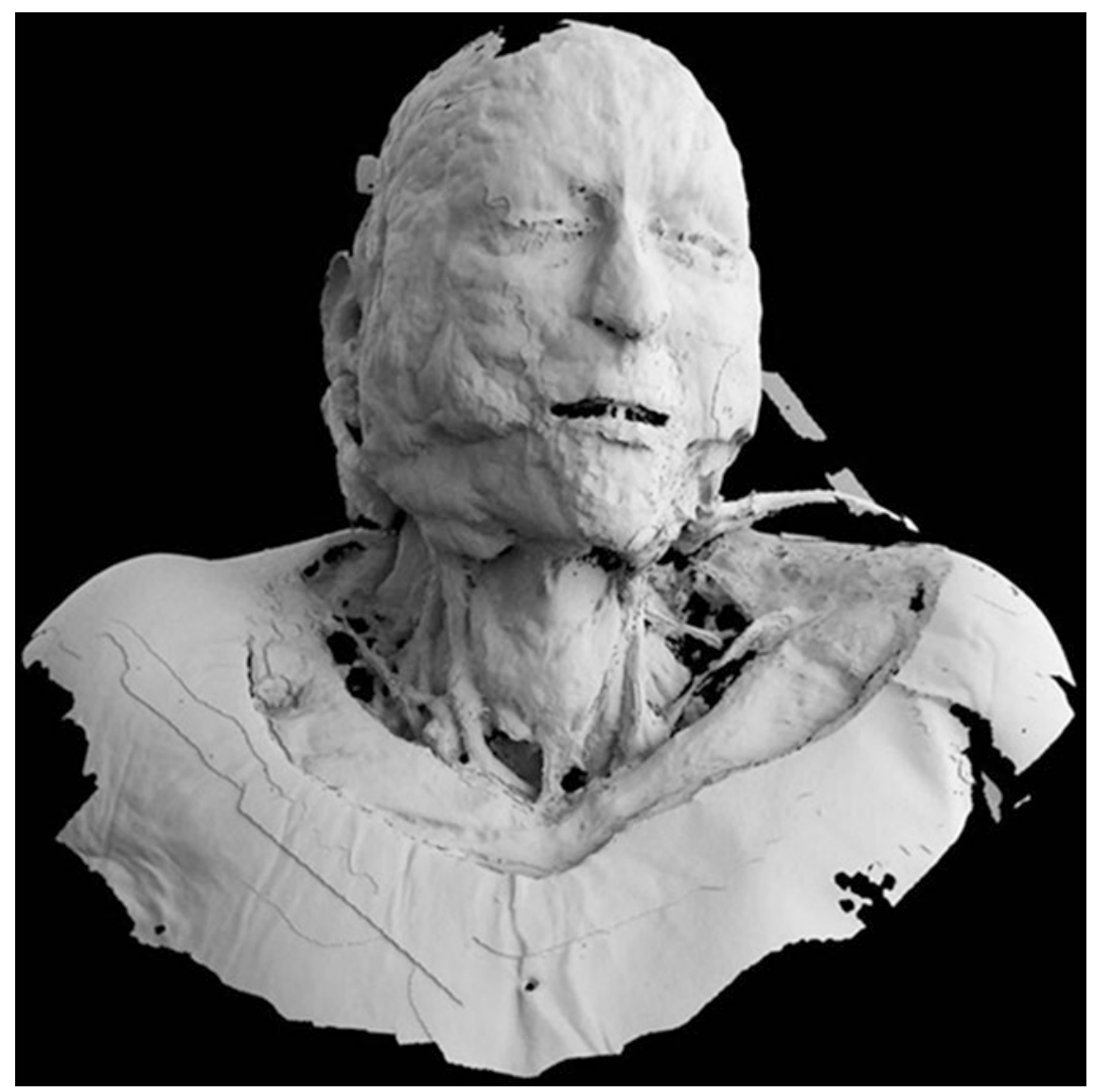

Fig. 3.3 Surface mesh generated from raw point cloud data

innervation to the upper limbs), and other closely related sensory and motor innervations. Also the larynx and trachea were included in soft tissue structure identification and data capture to clearly show anatomical relations for important clinical procedures, e.g. cricothyroidotomy, tracheostomy and airway intubation, as well as the oesophagus.

Following every stage of identification of all relevant anatomical structures in the head and neck, Perceptron Scanworks V5 laser scanning supported by 3D mesh processing software package, PolyWorks V12 (which aligns the partial scans and generates a mesh surface) were performed prior to the next, deeper dissection. (Figure 3.3 shows a polygon mesh model generated from raw highdensity $3 \mathrm{D}$ point clouds). This enabled a complete dataset to be recorded at all stages of dissection, which will then be able to be constructed and deconstructed by users as relevant to the training required, thus creating unique spatial awareness training. 


\subsubsection{Scanning of Skeletal Structures}

After soft tissue 3D data capturing, this was removed (apart from ocular structures) to demonstrate the skeletal structures of the head and neck including the neurocranium (calvaria and cranial base), viscerocranium (facial skeleton), mandible and vertebrae. This enables identification of all relevant foramina through which the cranial nerves exit/enter the skull and mandible. Perceptron Scanworks V5 3D laser scanner and PolyWorks were used again to capture the skeletal structures and to process the point cloud data.

Individual laser scans from multiple views of the skull were fused to create a high poly surface mesh, which is the only structure where generated mesh from scan data is directly used in the Head and Neck model. The geometric models of soft-tissue from scan data was found to not accurately represent their natural shape due to soft-tissue deformation resulting from gravity and realignment of the underlying bone structure. Therefore, soft tissue scan data and their mesh are used as a reference in the modelling process and all structures are validated by anatomy specialists to ensure accuracy.

\subsubsection{Intracranial Scanning}

At this stage, the vault of the skull and the brain were removed and the same laser scanning and 3D meshing were performed to record specifically all twelve pairs of cranial nerves, cerebral tissue, including parietal, frontal, temporal and occipital lobes with related gyri and sulci, and cerebral vasculature (e.g. Circle of Willis). Where the cranial nerves have been detached, repeat scanning of the base of the skull was undertaken to allow reconstruction of the full intracranial path of these nerves through the numerous foramina to their termination sites. At this stage the visual pathway was established using the gross anatomy of the optic nerves, optic chiasm, optic tracts with modelling of the lateral geniculate body combined with the previous capture of the midbrain and the occipital cortices. Intracranial vascular circulation was modelled based on standard anatomical and applied surgical knowledge.

After the base of the skull was scanned, the roof of the bony orbit was exposed to capture the extra-ocular muscles namely the levator palpebrae superioris, superior, inferior, lateral and medial recti, and the superior and inferior oblique incorporating their individual nerve supplies, and related vasculature and nerves surrounding these structures in each of the orbits. The cornea, anterior segment and retina were modelled based on existing anatomical knowledge and understanding.

\subsubsection{Photorealistic Texturing}

At each stage, the capture of 3D topographic data through the use of the Perceptron Scanworks V5 3D laser scanning was supported by high-resolution colour imaging. Since cadavers differ considerably in colour and texture from living tissue, and the 




Fig. 3.4 Texturing in Maya

shadow, specular highlights, and occlusions in photographs also make them not suitable for texture mapping [43], the photographic data of soft tissue were mainly used as references when building the geometry of models.

In order to produce a photorealistic and accurate model of the aforementioned structures, the skin surface, muscles and skeletal elements consist of several texture layers, describing colour, glossiness and surface structure subtleties. These were achieved through using a combination of photographs of living tissue, the poly-painting tool in Zbrush, and various other tools in Photoshop and Autodesk Maya. We produced visually realistic organ textures and appearances that are as close as possible the natural colour of skin and healthy living tissue. Figure 3.4 shows work-in-progress texturing in Maya.

\subsubsection{The Complete Dataset of Head and Neck}

The produced high resolution measured dataset is grouped and identified in Maya in order to serve a wide range of anatomical outputs. This enables the interface to present appropriately tagged data to the user, encapsulated in logical subsets. The tagging process includes clinically relevant, anatomically built structures that present context specific information on a case-by-case basis. A rendered head and neck model showing the muscle, nerve, and vascular layers is presented in Fig. 3.5. The resulting complete dataset does not represent any one specific human body but represents a comprehensive structure that captures and presents a normalised, unbiased anatomical model. 


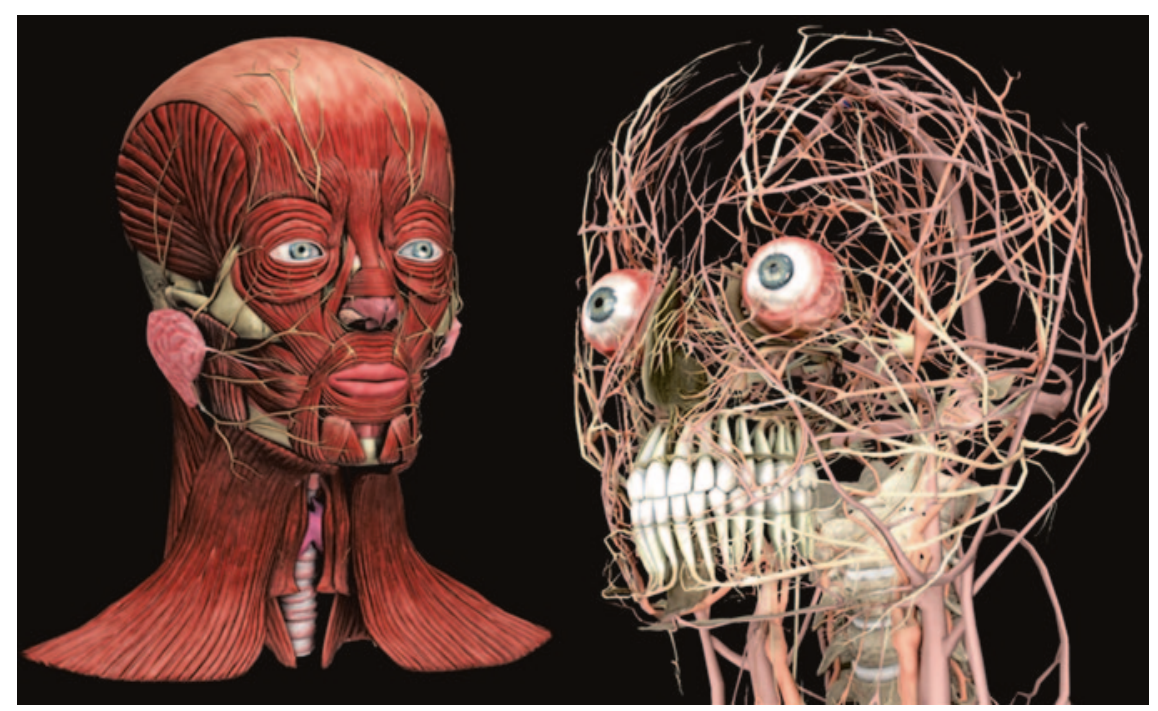

Fig. 3.5 Rendered head and neck showing the muscle, nerve, and vascular layers

\subsubsection{Interactive Application}

The uniqueness of the DDS model comes from forging together three key components:

- the anatomical precision and accuracy of the constructed datasets;

- the specialist clinical input to challenge and validate the model;

- The interface allowing proficient user-interactivity in real-time, enabling meaningful feedback and learning.

In order to use the head and neck model in a real-time application, low polygon models were created with normal maps baked from a Zbrush sculpt, decimating or re-topologizing in order to simplify the high polygon mesh.

Another importantly unique characteristic is the accompanying suite of manipulation tools, which afford both interactivity (for individual users) as well as interconnectivity (to support collaborative group usage). Figure 3.6 consists of a screenshot of the interactive Head and Neck Anatomy, showing a clipping plane on arbitrary angles and available functions in the vertical toolbar on the right. Apart from basic manipulation (such as object translation and rotation) and navigation controls (zoom, pan, etc.), it provides orthographic clipping planes as well as clipping plane on arbitrary angles. The virtual cutting planes reveal crosssection cuts which resembles cryosection or CT imaging.

The users can interact with the Head and Neck either through conventional input methods or through a combination of Xbox controller and head tracking. Together with passive stereoscopic projection, the latter interface provides an immersive experience for users to explore the internal structure of head and neck. 




Fig. 3.6 Screenshot of the interactive head and neck anatomy showing clipping plane on arbitrary angles and available features

The user can hide and reveal various anatomical structures, e.g. bones, muscles, nerves, vascular (Fig. 3.6-C), and conduct virtual dissection via a drag-and-drop function (Fig. 3.6-A). An 'explode' mode (Fig. 3.6-B) is available to allow the user to control an explosion via a slider, making the parts separate away from their original locations to reveal the inner details of the head and neck anatomy. Users can also save particular viewpoints and settings which could be loaded in the future (Fig. 3.6-D).

The interactive application also provides data annotation to support teaching and learning. Where clinically relevant, anatomically built structures are appropriately annotated with context specific information on a case-by-case basis to include text related content, linked diagrams and 3D images.

\subsubsection{Verification}

Working in collaboration with the 3D modelers in the team, every structure which had been dissected was validated during the modelling process, to ensure anatomical accuracy and a true likeness of the human body. The fidelity of the dataset was verified by the project's Clinical Advisory Board which comprised anatomists, clinicians and surgical specialists. Figure 3.7 shows an event of clinical validation. A senior clinical anatomist and government Licensed Teacher of Anatomy, ensured complete anatomical accuracy of all structures constructed in every stage. 


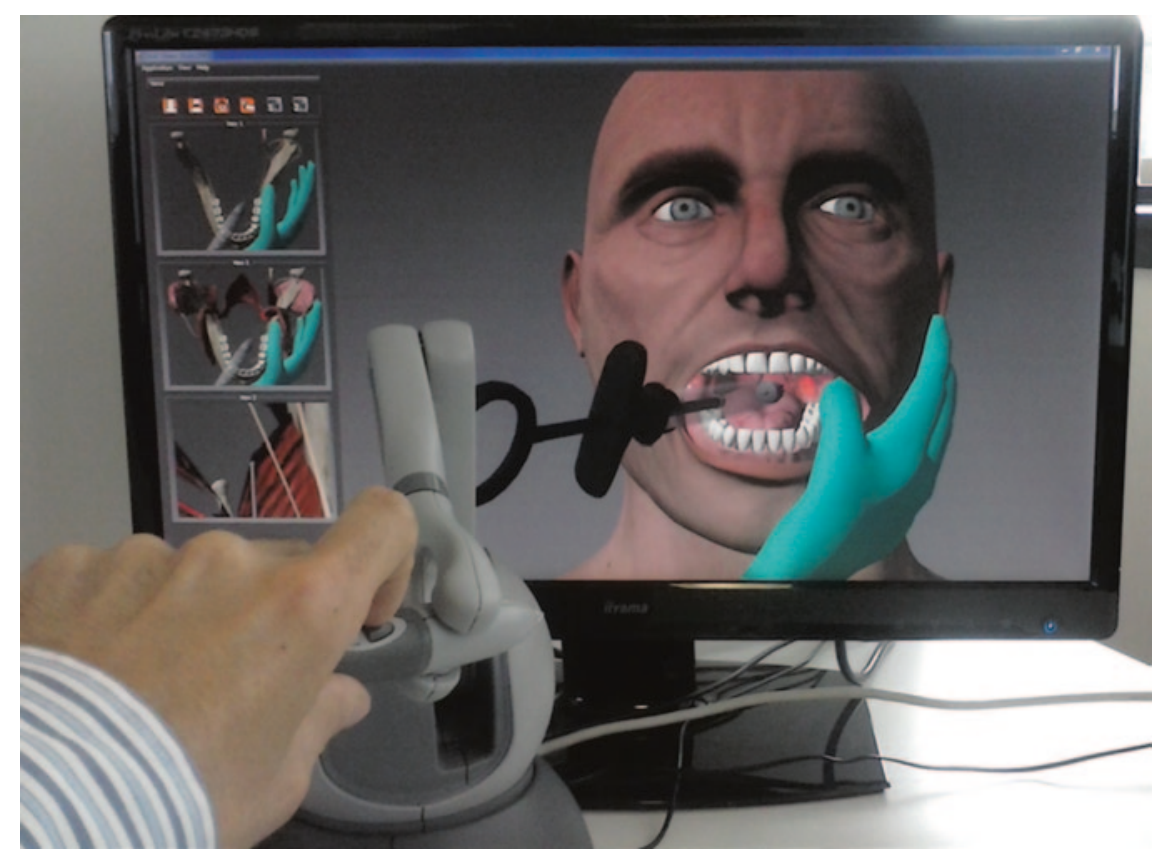

Fig. 3.7 The VR training system

The skull and mandible were initially reconstructed from the laser-captured skull. Then, a stringent schedule was created and adhered to at the beginning of the project for creating each and every musculoskeletal, vascular, neural and glandular structure. The approach undertaken was creating each anatomical structure from the deepest (closest to the skull) to the most superficial i.e. the skin (unlike the superficial to deep dissection). As every muscle, bone, nerve, blood vessel and glandular structure was created it had to be moulded around the framework of the skull. This involved using the laser scanned material and also the high resolution digital photography which was captured for all anatomical structures. This ensured direct correlation with every structure to the dissected, laser-scanned and photographed components.

All attachments, origins, terminations and pathway of every anatomical structure were meticulously created, where the anatomist and digital modelers would assign a set of structures to be modeled during a weekly period over the duration of the project. Therefore, this ensured that a catalogue of all anatomical structures in the head and neck was created which had to be modeled from the dissected material. On completion of the modelling of each anatomical structure, this was then reviewed as to its accuracy, initially of that individual element. As the work progressed and the model became more complex, each structure that was created had also to be examined in its accuracy to each and every surrounding anatomical structure. This ensured a completely anatomically correct and robust model was 
being developed. Where relevant, this was also examined by a clinician who operated in that field to ensure not just exceptionally accurate anatomical datasets were being created, but one with all relevant surgical anatomy clearly and accurately identifiable. This remained crucial where the anatomy of the oral cavity, including the teeth, had to be created to an exceptionally high level of accuracy never seen to date. This involved a team of oral surgeons and senior dental clinicians working side by side with the modelers during the duration of the project. As each anatomical area (e.g. floor of mouth, intracranial territory, orbit etc.) was created in the model, the modelers, anatomist, dental clinicians and surgeons then reviewed those set of structures in that territory and went through a rigorous "signing off" process when the work in that area was complete. To verify the accuracy of the model it was also externally examined and validated by senior academic clinicians specializing in head and neck anatomy. Again, this ensured that each and every anatomical structure gave a real-to-life representation of the structures, as well as ensuring the highest degree of accuracy of the anatomy created.

\subsection{Haptic Injection in the Head and Neck Model}

One of the most commonly performed procedure by dental practitioner is anaesthetizing the inferior alveolar nerve which runs from the angle of the mandible down the medial aspect of the mandible, innervating the lower teeth, lower lip, chin, and tongue. The successful development of procedural and psychomotor skills that are required for an efficient administration of local anesthesia is a crucial part of the dental curriculum [13]. The training for the psychomotor skill acquisition is traditionally carried out on anatomical models or live patients is critical $[35,44]$. Recently, the use of VR simulators has been shown to encourage the learning of sedative procedures in dental education [31]. Although, as mentioned previously in Sect. 3.2, VR simulations presented so far, do not enable the simulation of intrinsic haptic sensations perceived during the realization of the task in the real world. On the basis of outcomes from previous work $[6,8,17]$, the implementation of haptic force feedback in VR simulations is believed to enhance the learning of psychomotor skills involved in dental anesthesia injections.

In this section, we present the VR training system enhanced with haptic interaction, which is based on the Head and Neck model that we described in Sect. 3.3 and aims to support the successful development of psychomotor skills that are required in the anaesthetic procedure of the inferior alveolar nerve. The system displays the virtual anatomical head and neck model on which local anesthesia injections can be safely rehearsed aiming the performer to gain in proficiency and self-confidence prior to transition to first injections on live patients.

We use a Phantom Omni device, a cost effective solution for the simulation of realistic haptic force feedback in virtual environments. The Phantom Omni consists of a punctual inter-actuator able to sense position and orientation on 6 DOF input and render forces up to $3.3 \mathrm{~N}$ onto $3 \mathrm{DOF}$ output at $1 \mathrm{KHz}$ within a 
delimited workspace (up to $160 \mathrm{~W} \times 120 \mathrm{H} \times 70 \mathrm{D} \mathrm{mm}$ ). A dental syringe represented in the virtual environment is attached to the position and orientation of the stylus of the haptic device (Fig. 3.7). The contact of the needle on soft tissues and teeth, and corresponding haptic sensations (i.e. stiffness, damping, friction) can be perceived through the haptic device. Stiffness and damping force effects are respectively expressed as a function of the depth of penetration $x$ into the touched body and the velocity $v$ of the haptic device. These force effects consider the stiffness and damping properties of the touched body respectively expressed by the variable $k$ and $d$. These force effects allow defining the normal contact force perceived when the syringe tip is pressed onto a tissue (Eq. 3.1).

$$
F=-(k \cdot x-d \cdot v) \cdot N
$$

where $N$ is the normal reaction force to contact on the touched tissue.

Moreover, when that force exerted on a soft tissue overpasses a threshold, the syringe tip smoothly moves into the tissue and the haptic device simulates the puncture adding haptic sensations corresponding to all traversed tissues along a constraint line corresponding to the contrary normal vector at puncture point. The force effect corresponding to the motion of the syringe tip onto the surface of a soft tissue or a tooth is ruled the computation of friction effect. Friction encompasses static friction and kinematic friction. On the one hand, static friction describes how the body surface remains resistive to motion when no motion of the syringe tip has been engaged. On the other hand, dynamic friction describes how the body surface is resistive to the motion of the syringe tip once it has been engaged. Both friction effects refer to a resistive force to motion which magnitude can be expressed as a function of the normal reaction force to contact as in Eq. (3.2).

$$
F_{f}=\mu_{x} \cdot N
$$

where $\mu_{x}$ corresponds to the coefficient of friction of the surface of the touched soft tissue or tooth for each type of friction.

Buttons 1 and 2 of the haptic device can be used to respectively trigger and reset a graphical animation of the plunger pressing the anaesthetic solution for injection. Throughout an injection, the sedated area gradually turns to red (Fig. 3.7).

The VR training system allows assisting the performer while practicing by providing augmented information that could not be obtain throughout the training traditionally conducted in the real world. On the one hand, three additional views of the anatomical head and neck model can be provided through a panel located on the left side of the monitor (Fig. 3.7). The top view corresponds to a depurated view of the inferior mandible which includes the visualization of the inferior alveolar nerve. The middle view shows similar viewpoint but including the parotid glands, the buccinator, the superior pharyngeal constrictor and the medial pterygoid. The bottom view consists of a close-up on the tip of the needle. On the other hand, the system prompts real-time augmented information on performance outcomes in the form of warning messages which highlight a critical position of 
the needle in soft tissues (e.g. "Your needle is touching a nerve!", "Your needle is touching a blood vessel!", "Your needle is close to a gland!"). Moreover, the VR training system can also provide in real-time realistic audio augmented feedback which states for the mood of the patient when the needle insertion procedure becomes painful. According to behavioral psychologists, augmented information feedback is believed to play an important motivational role in the process of skill development [45].

\subsection{Conclusion and Future Work}

We have described the workflow of data construction, development, and validation of an interactive high-resolution three-dimensional anatomical model of head and neck with haptic interaction. The virtual Head and Neck represents a step change in anatomical construction, validation, visualization and interaction for viewing, teaching and dissemination. Head and neck anatomy is the most complex area of the entire human body. In this very small space, structures of the musculoskeletal, circulatory, lymphatic, nervous, endocrine and integumentary systems are tightly packed. In, for example, the musculoskeletal system, the smallest bones (middle ear: malleus, incus and stapes) and muscles (stapedius, being the smallest, and tensor tympani) of the body are tightly compacted into the petrous temporal bone. This compares starkly with, for example, the lower limb, which has the largest and strongest bone of the body (the femur), the longest muscle (sartorius), and the most powerful muscles (gluteal, quadriceps and hamstrings). With the circulatory system, the only blood supply to the brain passes through the very narrow region of the neck. Without these vessels, the brain would not be perfused, and life would not be compatible. Many other of the body's finest and most delicate structures are compacted into the head and neck territory. These include the muscles of facial expression, cranial nerves (supplying all head, neck and other structures), brain and spinal cord (and the complexity of the origins of spinal nerves) and bones thinner than paper (lamina papyracea of the ethmoid bone). What we have shown in this project is that even with the smallest structures of the whole body (found in the head and neck), we have managed to successfully acquire data digitally and reconstruct to the most accurate degree so far. As the rest of the body structures are much larger, more robust and easier to dissect, this will be able to be rolled out across all anatomical regions with ease.

In the long term, the results obtained from this three-year project can be viewed as the framework on which to build future efforts. Examples are: to expand models to whole body systems (a project creating a female breast model for breast cancer early detection has already begun); the inclusion of physiological-based animation/simulation; dynamic representations of the progression of diseases; dynamic simulation of deformable soft tissue and physiology, e.g. pulse, blood flow, hemodynamics and elasticity, for haptic display. 


\section{References}

1. Singare, S., Zhong, S., Xu, G., Wang, W., Zhou, J.: The use of laser scanner and rapid prototyping to fabricate auricular prosthesis. In: International Conference on E-Product E-Service and E-Entertainment (ICEEE 2010), pp. 1-3. doi:10.1109/ICEEE.2010.5661536 (2010)

2. Beveridge, E., Ma, M., Rea, P., Bale, K., Anderson, P.: 3D visualization for education, diagnosis and treatment of iliotibial band syndrome. In: Proceedings of the IEEE International Conference on Computer Medical Applications (ICCMA 2013), Sousse, Tunisia, 20-22 Jan 2013

3. Preim, B., Bartz, D.: Visualization in Medicine: Theory, Algorithms, and Applications. Morgan Kaufmann, Los Altos (2007)

4. Kalea, E.H., Mumcuoglua, E.U., Hamcanb, S.: Automatic segmentation of human facial tissue by MRI-CT fusion: A feasibility study. Comput. Meth. Programs Biomed. 108(3), 1106-1120 (2012). Elsevier

5. RCUK and Universities UK. Big Ideas for the Future. June 2012. Available from. http://www.rcuk.ac.uk/Publications/reports/Pages/BigIdeas.aspx. Accessed 13 May 2013

6. Xia, P., Lopes, A.M., Restivo, M.T.: Virtual reality and haptics for dental surgery: a personal review. Vis. Comput. 29(5), 433-447 (2013)

7. Steinberg, A.D., Bashook, P.G., Drummond, J., Ashrafi, S., Zefran, M.: Assessment of faculty perception of content validity of Periosim $\odot$, a haptic-3D virtual reality dental training simulator. J. Dent. Educ. 71(12), 1574-1582 (2007)

8. Dutã, M., Amariei, C.I., Bogdan, C.M., Popovici, D.M., Ionescu, N., Nuca, C.I.: An overview of virtual and augmented reality in dental education. Oral Health Dent Manage. 10, 1 (2011)

9. Kim, L., Hwang, Y., Park, S.H., Ha, S.: Dental training system using multi-modal interface. Comput. Aided Des. Appl. 2(5), 591-598 (2005)

10. Rhienmora, P., Haddawy, P., Dailey, M.N., Khanal, P., Suebnukarn, S.: Development of a dental skills training simulator using virtual reality and haptic device. NECTEC Tech. J. 8(20), 140-147 (2008)

11. Konukseven, E.I., Önder, M.E., Mumcuoglu, E., Kisnisci, R.S.: Development of a visio-haptic integrated dental training simulation system. J. Dent. Educ. 74(8), 880-891 (2010)

12. Tse, B., Harwin, W., Barrow, A., Quinn, B., Cox, M.: Design and development of a haptic dental training system-hapTEL. Haptics: Generating and Perceiving Tangible Sensations, pp. 101-108. Springer, Berlin Heidelberg (2010)

13. Brand, H.S., Kuin, D., Baart, J.A.: A survey of local anaesthesia education in European dental schools. Eur. J. Dent. Educ. 12(2), 85-88 (2008)

14. Brand, H.S., Tan, L.L.S., van der Spek, S.J., Baart, J.A.: European dental students opinions on their local anaesthesia education. Eur. J. Dent. Educ. 15(1), 47-52 (2011)

15. Taylor, C.L., Grey, N., Satterthwaite, J.D.: Assessing the clinical skills of dental students: a review of the literature. J. Educ. Learn. 2(1), 20 (2013)

16. Luciano, C., Banerjee, P., DeFanti, T.: Haptics-based virtual reality periodontal training simulator. Virtual Reality 13(2), 69-85 (2009)

17. Cox, M.J., Quinn, B.F., Newton, J.T., Banerjee, A., Woolford, M.: Researching haptics in higher education: The complexity of developing haptics virtual learning systems and evaluating its impact on students learning. Comput. Educ. 59(1), 156-166 (2012)

18. Gottlieb, R., Vervoorn, J.M., Buchanan, J.: Simulation in dentistry and oral health in the comprehensive textbook of healthcare simulation chapter 21, pp. 329-340. Springer, New York (2013)

19. Noborio, H., Sasaki, D., Kawamoto, Y., Tatsumi, T., Sohmura, T.: Mixed reality software for dental simulation system. In: IEEE International Workshop on Haptic Audio visual Environments and Games. HAVE 2008, pp. 19-24. IEEE (2008)

20. Yoshida, Y., Yamaguchi, S., Kawamoto, Y., Noborio, H., Murakami, S., Sohmura, T.: Development of a multi-layered virtual tooth model for the haptic dental training system. Dent. Mater. J. 30(1), 1-6 (2011) 
21. Forsslund, J., Lund, B., Sallnäs Pysander, E., Rosen, A.: Towards an Oral Surgery Simulator. World Dental Congress, Stockholm. http://www.forsslundsystems.com/images/documents/forsslund_ et_al_fdi_2008_poster.pdf (2008)

22. Lund, B., Fors, U., Sejersen, R., Sallnäs, E.L., Rosén, A.: Student perception of two different simulation techniques in oral and maxillofacial surgery undergraduate training. BMC Med. Educ. 11(1), 82 (2011)

23. Kolesnikov, M., Zefran, M., Steinberg, A.D., Bashook, P.G.: PerioSim: Haptic virtual reality simulator for sensorimotor skill acquisition in dentistry. In: IEEE International Conference on Robotics and Automation. ICRA'09, pp. 689-694. IEEE (2009)

24. Pohlenz, P., Gröbe, A., Petersik, A., Von Sternberg, N., Pflesser, B., Pommert, A., Heiland, M.: Virtual dental surgery as a new educational tool in dental school. J. Cranio-Maxillofac. Surg. 38(8), 560-564 (2010)

25. Suebnukarn, S., Haddawy, P., Rhienmora, P., Jittimanee, P., Viratket, P.: Augmented kinematic feedback from haptic virtual reality for dental skill acquisition. J. Dent. Educ. 74(12), 1357-1366 (2010)

26. Rhienmora, P., Haddawy, P., Suebnukarn, S., Dailey, M.N.: Intelligent dental training simulator with objective skill assessment and feedback. Artif. Intell. Med. 52(2), 115-121 (2011)

27. Rhienmora, P., Gajananan, K., Haddawy, P., Dailey, M.N., Suebnukarn, S.: Augmented reality haptics system for dental surgical skills training. In: Proceedings of the 17th ACM Symposium on Virtual Reality Software and Technology, pp. 97-98. ACM (2010)

28. Bakker, D., Lagerweij, M., Wesselink, P., Vervoorn, M.: Transfer of manual dexterity skills acquired in the Simodont, a dental haptic trainer with a virtual environment, to reality: a pilot study. Bio-Algorithms Med-Syst. 6(11), 21-24 (2010)

29. Corneliu, A., Mihaela, D., Mircea-Dorin, P., Crenguta, B., Mircea, G.: Teeth reduction dental preparation using virtual and augmented reality by Constanta dental medicine students through the VirDenT system. In: The International Conference Development, Energy, Environment, Economics, Puerto De La Cruz, Tenerife, Spain (2011)

30. Wang, D., Zhang, Y., Hou, J., Wang, Y., Lv, P., Chen, Y., Zhao, H.: iDental: a haptic-based dental simulator and its preliminary user evaluation. IEEE Trans. Haptics 5, 4 (2012)

31. Hanson, K.M.: The utilization of mixed-reality technologies to teach techniques for administering local anesthesia. PhD thesis, Utah State University, Logan, Utah (2011)

32. Coles, T.R., Meglan, D., John, N.W.: The role of haptics in medical training simulators: a survey of the state of the art. IEEE Trans. Haptics 4(1), 51-66 (2011)

33. Riener, R., Harders, M.: VR for Medical Training in Virtual Reality in Medicine, pp. 181-210. Springer, London (2012)

34. Hanson, K.M, Jones, N, Krantz, M, Law, H.: Techniques for administering local anesthesia utilizing mixed-reality technology. J. Dent. Res. 85, 1693 (2006). https://iadr.confex.com/iadr /2006Orld/techprogramforcd/A77559.htm

35. Brand, H.S., Baart, J.A., Maas, N.E., Bachet, I.: Effect of a training model in local anesthesia teaching. J. Dent. Educ. 74(8), 876-879 (2010)

36. Utting, M., Willan, P.: What future for dissection in courses of human topographical anatomy in universities in the UK. Clin. Anat. 8, 414-417 (1995)

37. Dangerfield, P., Bradley, P., Gibbs, T.: Learning gross anatomy in a clinical skills course. Clin. Anat. 2000(13), 444-447 (2000)

38. Fitzgerald, J.E., White, M.J., Tang, S.W., Maxwell-Armstrong, C.A., James, D.K.: Are we teaching sufficient anatomy at medical school? The opinions of newly qualified doctors. Clin. Anat. 21, 718-724 (2008)

39. Patel, K.M., Moxham, B.J.: Attitudes of professional anatomists to curricular change. Clin. Anat. 2006(19), 132-141 (2006)

40. Ma, M., Bale, K., Rea, P.: Constructionist learning in anatomy education: what anatomy students can learn through serious games development. In: Ma M., Oliveira M., Baalsrud Hauge J., Duin H., Thoben K. (eds.) Serious Games Development and Applications, Lecture Notes in Computer Science, LNCS, vol. 7528, pp. 43-58. Springer, Berlin Heidelberg (2012) 
41. Sugand, K., Abrahams, P., Khurana, A.: The anatomy of anatomy: a review for its modernization. Anat. Sci. Educ. 3(2), 83-93 (2010)

42. Turney, B.W.: Anatomy in a modern medical curriculum. Ann. R. Coll. Surg. Engl. 89, 104-107 (2007)

43. Cenydd, L., John, N.W., Bloj, M., Walter, A., Phillips, N.I.: Visualizing the surface of a living human brain. IEEE Comput. Graph. Appl. 32(2), 55-65 (2012)

44. Tomruk, C.Ö., Oktay, I., Şençift, K.: A survey of local anesthesia education in Turkish dental schools. J. Dent. Educ. 77(3), 348-350 (2013)

45. Schmidt, R.A., Wrisberg, C.A.: Motor Learning and Performance: A Situation-Based Learning Approach, 4th edn. Human Kinetics Publishers, Champaign, IL (2008) 